\title{
OPEN Association between sanitary toilet coverage rate and intestinal infectious disease in Jiangsu Province, China
}

\author{
TingTing Chen \& Kraiwuth Kallawicha ${ }^{\bowtie}$
}

Intestinal infectious disease is one of the most common diseases in China and is prevalent worldwide. The Chinese government launched a toilet improvement campaign to improve sanitation and reduce the incidence of diseases. This study determined the association between sanitary toilet use and intestinal infectious disease incidence in Jiangsu Province, China during 2011-2019. This study adopted an ecological retrospective research design. All secondary data were obtained through government websites and government information disclosure channels. Multiple linear regression was employed to analyze the association between the incidence of intestinal infectious diseases and sanitary toilet coverage rate and other potential predictors. Data suggested that the aggregate annual incidence of Type $A$ and $B$ intestinal infectious diseases showed a downward trend, the aggregate annual incidence of other infectious diarrhea continued to increase, and hand-foot-mouth disease occurred every other year with the highest annual incidence rate. The incidence was higher in coastal cities. Multiple regression results indicated that the usage of three types of sanitary toilets, compliance rate of water quality, and average ambient temperature have an impact on intestinal infectious diseases. The aggregate annual incidence of Type $A$ and $B$ intestinal infectious disease was negatively correlated with the cumulative use of sanitary toilets $(B=-0.036)$ and surface water quality $(b=-0.135 ; p<0.05)$. Increase in sanitary toilet use and water quality control can reduce the number of new cases, which will be beneficial for the population in the province. Moreover, the toilet improvement interventions should continue to maintain high-quality construction.

Intestinal infectious diseases are one of the most common diseases prevalent worldwide. These diseases place a significant health burden on individuals and communities at large, and the morbidity resulting from these diseases are high even in developed countries ${ }^{1}$. A study indicated that intestinal infectious diseases affect approximately $25 \%$ of the UK population annually, resulting in a damage of approximately 1.5 billion pounds to the economy, population, and the National Health Service each year ${ }^{2}$. Furthermore, intestinal infectious diseases are the leading cause of death in South Asian countries ${ }^{3}$. Many studies have reported the cause of these diseases including consumption of microbe-contaminated food, lack of clean water, or high levels of poverty ${ }^{4-6}$. One of the crucial problems that lead to high prevalence of these diseases is the lack of sanitary toilets and poor hygienic conditions ${ }^{7}$. These impediments to public health have been observed worldwide, particularly in rural areas.

Because of inadequate toilet sanitation, human feces are directly exposed to air, in turn providing a favorable environment for the breeding of mosquitoes and flies. In addition, because of improper cleaning of feces, many parasites and bacteria present in the feces spread through soil and water sources and become the main source of various diseases, particularly intestinal infectious diseases. Some studies have highlighted that poor toilet hygiene is a risk factor for intestinal infectious diseases; however, the association between sanitary toilets and intestinal infectious diseases has not been supported by any direct evidence ${ }^{8,9}$. The World Bank estimates that inadequate sanitation and unsafe discharge of toilet water results in approximately 1.5 million children getting infected with intestinal infections each year ${ }^{10}$. Furthermore, many studies in developing countries have reported that limited access to toilets can increase intestinal infectious disease incidence with children being the most vulnerable group ${ }^{11-13}$. 
China is among the countries affected by high intestinal infectious disease prevalence, and its incidence is especially conspicuous in rural areas ${ }^{14}$. The practice of collecting human waste for the purpose of agricultural fertilization since ancient times has been the source of toilet hygiene problems in China. The prevention and control of diseases did not have a smooth implementation, resulting in the incidence of intestinal infectious diseases being markedly high. This health crisis has received more attention after the founding of the People's Republic of China ${ }^{15}$. In 1950s, because of the poor conditions of urban and rural environments and the threat of germ warfare in China, the Patriotic Health Campaign was launched nationwide, from China's toilet improvement initiative originated ${ }^{16}$. The initiative gradually gained momentum through numerous national campaigns, and the pilot construction of rural sanitary toilets began occurring throughout the country. In 2015, the government began paying more attention to toilet improvement, as per the instruction of the General Secretary, Xi Jinping, and the campaign was extended to vast rural areas ${ }^{17}$. However, approximately 17 million households in China still face serious sanitation problems because of poor toilet conditions. Previous evidence has suggested that only $48.6 \%$ of rural households have access to sanitary toilets, while $2 \%$ still do not have toilets ${ }^{18}$. This problem of limited accessibility to toilets can lead to the spreading of intestinal infectious diseases.

Jiangsu Province has been actively implementing the national health work policy, and it ranks first in terms of health promotion. The rural toilet improvement in Jiangsu Province has been at the forefront of the country's toilet improvement initiative, leading to the popularity of sanitary toilets growing rapidly in the province ${ }^{16}$. However, the latest data released in 2019 show that intestinal infectious diseases are still among the common infectious diseases affecting Jiangsu Province, accounting for approximately $45 \%$ of all mandatory reported infectious diseases ${ }^{19}$.

To our knowledge, limited attention has been paid to the improvement of toilets and intestinal infectious diseases in Jiangsu Province. Therefore, in this study, we investigated the association between the increase in sanitary toilets and the incidence of intestinal infectious diseases in Jiangsu Province of China during 2011-2019. In addition, water resource quality, vector density, population density and other environmental factors were considered in the analysis.

\section{Materials and methods}

Study design. This study employed an ecological retrospective design to study the association between the increase of sanitary toilets and intestinal infectious diseases between 2011 and 2019 in Jiangsu Province. This province is located on the eastern coast of China with a total population of 80.7 million as of 2019 . The analysis of this study was conducted at two levels. First, Jiangsu Province was considered a single unit. Second, 13 prefecture-level cities (i.e., Nanjing, Wuxi, Xuzhou, Changzhou, Suzhou, Nantong, Lianyungang, Huaian, Yancheng, Yangzhou, Zhenjiang, Taizhou, and Suqian) of Jiangsu Province were analyzed separately as the second level.

Ethical review. The study protocol followed the Declaration of Helsinki of 1975, as revised in 1983. Secondary data were obtained in the province and city levels. None of individual data were exposed or used in the analysis. Therefore, the informed consent was waived. The study protocol was approved and the informed consent was waived by the Research Ethics Review Committee for Research involving Human Research Participants, Group I, Chulalongkorn University (Ethic No. 102/2020).

Data collection. Dependent variable (intestinal infectious diseases). Intestinal infectious diseases were analyzed in this study on the basis of mandatory reported infectious diseases in China. Three major types (six diseases) of intestinal infectious diseases were studied: Type A, which included cholera (ICD-10-A00); Type B, which included typhoid (i.e., typhoid [ICD-10-A01.0] and paratyphoid [ICD-10-A01.1-A01.4]), dysentery (i.e., bacillary dysentery [ICD-10-A03.0] and amoebic dysentery [ICD-10-A06.0]), and viral hepatitis (i.e. hepatitis A [ICD-10-B15.], hepatitis E [ICD-10-B17.2], and untyped hepatitis [ICD-10-B19]); and Type C, which included other infectious diarrhea (i.e., infectious diarrhea other than cholera, bacterial and amoebic dysentery, and typhoid fever and paratyphoid fever [ICD-10-A09]) and hand-foot-mouth disease (HFMD) [ICD-10-B08.4]. The characteristics of each infectious disease studied here, including types of pathogens, modes of transmission, and average incidence, are presented in Supplement material Table S1. These data were retrieved from the Jiangsu Provincial Health Commission through official websites and government information disclosure applications.

The aggregate annual incidence of all intestinal infectious diseases (three types) for each year was calculated as:

$$
\text { Aggregate annual incidence }=\frac{\text { Total no. of new cases of diseases reported each year }}{\text { Total population of Jiangsu Province }(\text { at risk) }} \times 100,000,
$$

whereas the annual incidence of each disease was calculated as:

$$
\text { Annual incidence }=\frac{\text { No. of new cases of each disease reported each year }}{\text { Total population of Jiangsu Province (at risk) }} \times 100,000
$$

Independent variables. In this study, the term sanitary toilet refers to types of toilets, namely, sanitary toilets, harmless sanitary toilets, and sanitary public toilets. Toilets with fences and roofs and an impermeable and airtight septic tank for waste collection were defined as sanitary toilets. These toilets are clean, free of fly maggots, and odorless. Moreover, feces is removed as and when required. Harmless sanitary toilets referred to those that met the basic requirements of sanitary toilets. In addition, these toilets have facilities for the decontamination 


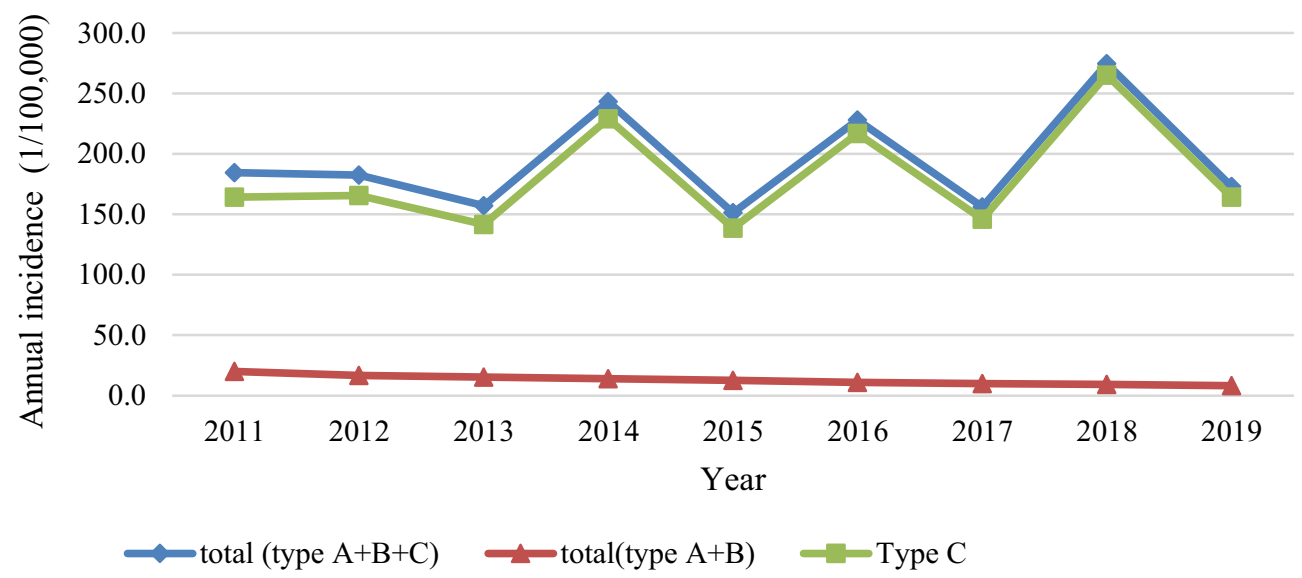

Figure 1. Variation trends in three types of intestinal infectious diseases in Jiangsu Province during 2011-2019.

of feces and are managed according to specification. Finally, public sanitary toilets referred to the ones that are available in public areas for those who do not have toilets available at home. The structure of each type of toilet is provided in Supplement materials Fig. S1. These data were retrieved from the China Statistical Yearbook on Environment. The sanitary toilet coverage rate was calculated as follows:

$$
\text { Sanitary toilet coverage rate }=\frac{\text { No. of households using various types of sanitary toilets }}{\text { Total no. of rural households }} \times 100
$$

Information on surface water quality was obtained from the cross-sectional data from the national surface water environmental quality assessment conducted by the Water Resources Bureau. The average annual water quality that met the Class III standard (i.e., water that qualified for further processing as drinking water) was included for the analysis. The compliance rate of water quality was calculated as follows:

$$
\text { Compliance rate }=\frac{\text { No. of qualified samples }}{\text { Total no. of samples }} \times 100 \%
$$

Vector density (i.e., mice, flies, cockroaches, and mosquitoes) was also collected at select monitoring points according to different geographic locations. Data were retrieved from the Department of Ecology and Environment of Jiangsu Province. The density was calculated as follows:

$$
\text { Vector density }=\frac{\text { No. of vectors }}{\text { No. of traps }}
$$

Rainfall $(\mathrm{mm})$ and temperature $\left({ }^{\circ} \mathrm{C}\right)$ comprised other environmental parameters. Temperature data comprised the annual average temperature and maximum and minimum temperature. These data were retrieved from the Jiangsu Statistical Yearbook.

Statistical analysis. The distribution of the coverage rate of sanitary toilets in Jiangsu Province and each prefecture-level city from 2011 to 2019 (Eq. 3), trend of the incidence of intestinal infectious diseases (Eqs. 1 and 2), and other independent variables (Eqs. 4 and 5) were described using descriptive statistics. The association between the incidence of three types and each intestinal infectious disease, sanitary toilet coverage rate, and other potential predictor variables were prescreened using a simple linear regression analysis. Independent variables that reached a significance level of $p<0.2$ were considered for further multiple regression analysis. Independent variables with $p<0.05$ were considered statistically significant in the final multiple regression model. In addition, the city-specific models were analyzed. However, because of the lack of toilet coverage information in each city, only environmental parameters were included in the analysis. All statistical analyses were performed using IBM SPSS Statistics for Windows (version 22; IBM, Armonk, NY, USA). ArcGIS (version 10.6; Esri, Redlands, CA, USA) was used to illustrate the spatial distribution of the disease incidence.

Ethical approval. The study protocol was approved and the informed consent was waived by the Research Ethics Review Committee for Research involving Human Research Participants, Group I, Chulalongkorn University (Ethic No. 102/2020).

\section{Results}

Distribution of intestinal infectious diseases in Jiangsu Province. According to the data from 2011 to 2019 presented in Fig. 1, Type C intestinal infectious disease was the most common type of disease reported to the government, whereas Type A and B intestinal infectious diseases were far less observed. The distribution patterns of Type A and B intestinal infectious diseases showed a clear downward trend, from 20.04 per 100,000 


\begin{tabular}{|c|c|c|c|c|c|c|c|}
\hline \multicolumn{8}{|c|}{ Incidence rate of intestinal infectious disease $(1 / 100,000)^{a}$} \\
\hline \multirow[b]{2}{*}{ Year } & \multirow{2}{*}{\begin{tabular}{|l|} 
Type A \\
Cholera
\end{tabular}} & \multicolumn{3}{|l|}{ Type B } & \multicolumn{2}{|l|}{ Type C } & \multirow[b]{2}{*}{ Total } \\
\hline & & Viral hepatitis & Dysentery & Typhoid, paratyphoid & $\begin{array}{l}\text { Other infectious } \\
\text { diarrhea }\end{array}$ & $\begin{array}{l}\text { Hand-foot-mouth } \\
\text { disease }\end{array}$ & \\
\hline 2011 & 0.0 & 10.3 & 9.4 & 0.3 & 18.3 & 146.0 & 184.3 \\
\hline 2012 & 0.0 & 9.3 & 7.3 & 0.3 & 19.2 & 146.5 & 182.5 \\
\hline 2013 & 0.0 & 9.5 & 5.7 & 0.3 & 20.1 & 121.7 & 157.3 \\
\hline 2014 & 0.0 & 8.8 & 5.0 & 0.2 & 18.7 & 210.5 & 243.2 \\
\hline 2015 & \begin{tabular}{|l|} 
\\
\end{tabular} & 7.6 & \begin{tabular}{|l|}
4.9 \\
\end{tabular} & 0.2 & \begin{tabular}{|l|l|}
19.2 \\
\end{tabular} & \begin{tabular}{|l|l}
119.4 \\
\end{tabular} & 151.3 \\
\hline 2016 & 0.0 & 6.7 & 4.1 & 0.4 & \begin{tabular}{|l|l|}
19.3 \\
\end{tabular} & \begin{tabular}{|l|}
197.6 \\
\end{tabular} & 228.0 \\
\hline 2017 & 0.0 & 6.4 & 3.4 & 0.2 & \begin{tabular}{|l|l|}
31.9 \\
\end{tabular} & \begin{tabular}{|l|l|}
114.2 \\
\end{tabular} & 156.1 \\
\hline 2018 & \begin{tabular}{|l|}
0.0 \\
\end{tabular} & 6.0 & \begin{tabular}{|l|l}
3.1 \\
\end{tabular} & 0.2 & 27.9 & 237.5 & \begin{tabular}{|l|l}
274.6 \\
\end{tabular} \\
\hline 2019 & \begin{tabular}{|l|}
0.0 \\
\end{tabular} & 5.9 & 2.8 & 0.2 & \begin{tabular}{|l|l|}
33.5 \\
\end{tabular} & \begin{tabular}{|l|l}
130.7 \\
\end{tabular} & \begin{tabular}{|l|l}
173.0 \\
\end{tabular} \\
\hline Mean & 0.0 & 7.8 & 5.1 & 0.3 & 23.1 & \begin{tabular}{|l|l}
158.2 \\
\end{tabular} & 194.5 \\
\hline
\end{tabular}

Table 1. Annual incidence rate of each intestinal infectious disease in Jiangsu Province for each year during 2011-2019. The permanent residents of Jiangsu Province in 2019 amounted to 80.7 million. ${ }^{\text {a Annual incidence }}$ rates were calculated as Eq. (2).

in 2011 to 8.28 per 100,000 in 2019 (Eq. 2), whereas the pattern of Type C intestinal infectious diseases fluctuated with a peak incidence every other year. The distribution pattern clearly indicated that the aggregate annual incidence of three types of diseases (Eq. 1) was generally consistent with that of Type $C$ intestinal infectious diseases.

As demonstrated in Table 1, the annual incidence of Type A intestinal infectious disease was very low. This is because of the very large denominator (total population of Jiangsu Province) compared to the numerator (annual case number) (Eq. 2). Therefore, we combined the annual incidence of Type A and B intestinal infectious diseases for further statistical analysis (Eq. 1). The annual incidence of HFMD was the highest among all studied diseases, accounting for $81 \%$ of the total intestinal infectious diseases. Its average incidence in 9 years was 158.2 per 100,000. Other infectious diarrhea had the second highest annual incidence, accounting for $12 \%$ of the total intestinal infectious diseases. The annual incidence of viral hepatitis and dysentery accounted for $4 \%$ and $3 \%$. The annual incidence of typhoid and paratyphoid and cholera were very low. The distribution of viral hepatitis and dysentery annual incidence decreased over the study period, while the annual incidence of other infectious diarrhea generally increased. Moreover, the fluctuating annual incidence pattern of HFMD, which peaked every other year, showed a similar trend to the aggregate annual incidence of three types of intestinal infectious diseases.

Figures 2 and 3 demonstrate the spatial and temporal distribution of the average aggregate annual incidence of Type A and B intestinal infectious diseases in Jiangsu Province from 2011 to 2019, respectively. In Fig. 2, the dot density map shows the average aggregate annual incidence where one dot refers to $1 / 100,000$ case. In general, the inner cities had lower annual incidence rate compared with the middle and southern cities. The lowest annual incidence was observed in Huai'an city while the highest annual incidence was observed in Xuzhou city. As presented in Fig. 3, the temporal distribution of diseases in each city showed a downward trend from 2011 to 2019, except in Xuzhou city where a reverse pattern was observed since 2016, inconsistent with the patterns in other cities.

Distribution of toilet coverage rate and environmental parameters. Figure 4 presents the trends in the cumulative number of three types of toilets from 2011 to 2019 (Eq. 3). The number of each specific type of toilet increased year after year after the government implemented the campaign. The coverage rate of harmless sanitary toilets showed the most change, increasing from approximately 10 million households in 2011 to 15.4 million households in 2019.

Table 2 highlights the vector density and other environmental data analyzed during the study period (Eqs. 4 and 5). Data on the vector density were available from 2011 to 2018. No consistent pattern of change was observed in the overall density of the four pests in Jiangsu Province. The coverage rate of Class III water sources showed an increasing pattern from $36 \%$ in 2011 to $78 \%$ in 2019 , implying that the quality of surface water was consistently improved during the entire study period. Similarly, the average temperature in Jiangsu Province showed a slight increase in the past 9 years, whereas rainfall varied across the study period, reaching $1612.3 \mathrm{~mm}$ in 2016.

Association between intestinal infectious diseases and other variables. Table 3 presents the results of the simple regression analysis of intestinal infectious diseases and potential predictor variables $(p<0.2)$. As expected, the number of households using sanitary toilets and the average temperatures showed a negative association with incidence of diseases. Similarly, the surface water quality and temperature were negatively associated with the incidence of diseases. These variables were further analyzed using multiple regression.

In the final multiple regression analysis, two models remained significant (Table 4 ). The results highlighted that the cumulative number of households using sanitary toilets $(\beta=-0.036)$ and the compliance rate of surface water $(\beta=-0.135)$ were negatively associated with the aggregate annual incidence of type A and B intestinal 
- prefecture-level city administrative region of a city 1 dot $=1 / 100000$ case

- incidence

Figure 2. Distribution of the average aggregate annual incidence of Type A and B intestinal infectious diseases in Jiangsu Province during 2011-2019 (Map was created using ArcGIS version 10.6; https://desktop.arcgis.com/ en/).

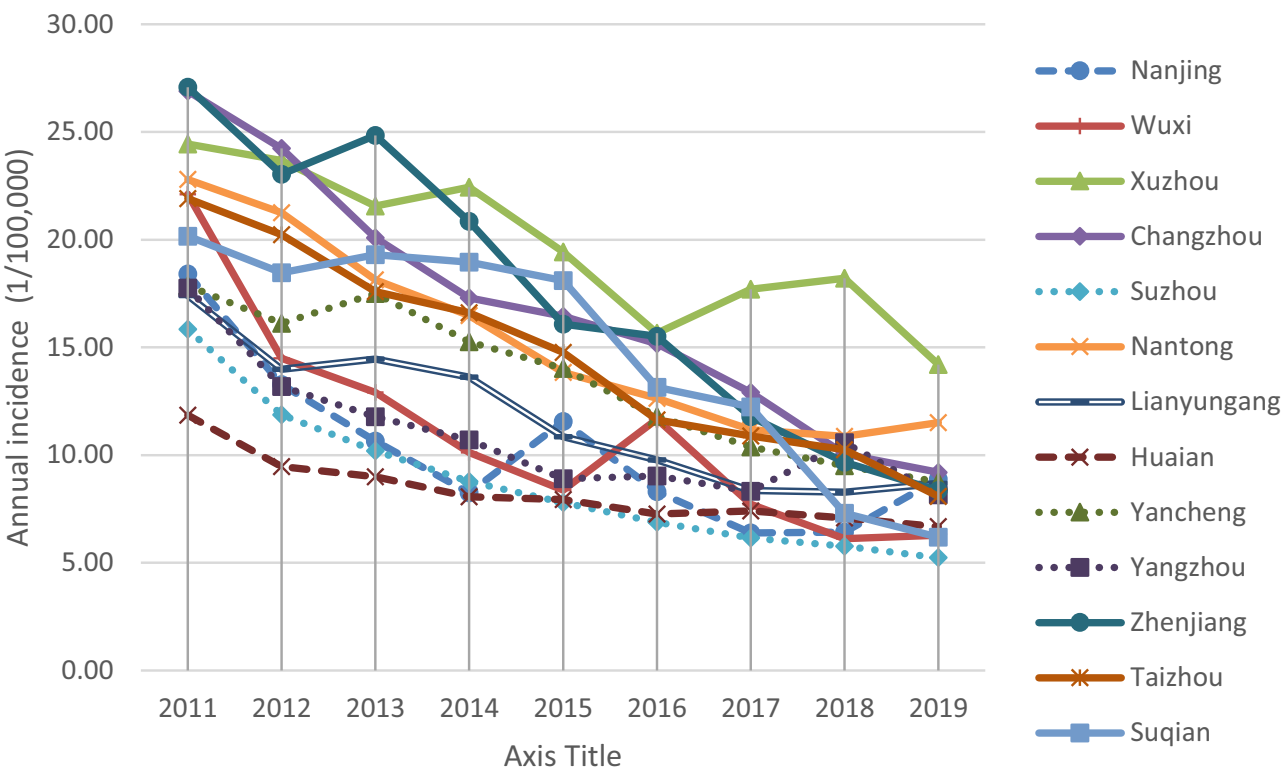

Figure 3. Trends in the average aggregate annual incidence of Type A and B intestinal infectious diseases in 13 cities of Jiangsu Province.

infectious diseases in Jiangsu Province $\left(R^{2}=0.996, p<0.05\right)$. According to the analysis, the incidence of Type A and $\mathrm{B}$ intestinal infectious diseases were aggregated and treated as dependent variable because of the very low annual incidence of Type A. Therefore, the association observed in this part was rather for Type B intestinal infectious disease. Moreover, the results indicated that the cumulative number of sanitary toilets used $(\beta=-0.016)$ and sanitary public toilets used $(\beta=-0.159)$ were negatively associated with the annual incidence of dysentery $\left(R^{2}=0.998, p<0.05\right)$. 


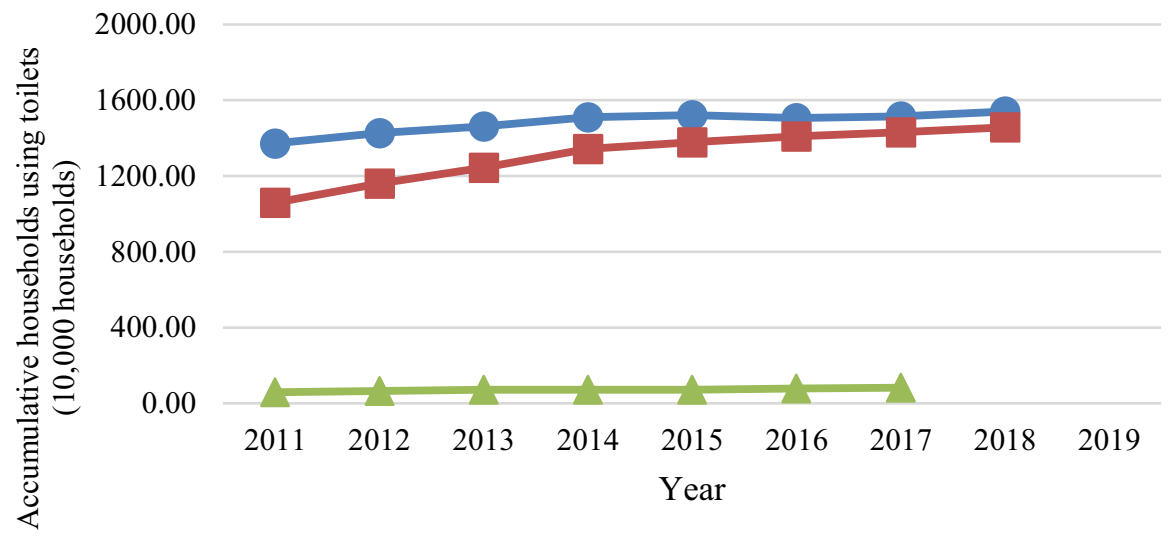

- sanitary toliet $\longrightarrow$ harmless sanitary toilet $\longrightarrow$ sanitary public toilet

Figure 4. Trends in the cumulative households using three types of sanitary toilets (Unit $=10,000$ households).

\begin{tabular}{|l|l|l|l|l|l|l|l|}
\hline \multirow{2}{*}{ Year } & \multicolumn{3}{|l|}{ Vector density (Eq. 5) } & \multicolumn{2}{|l|}{} & \\
\cline { 2 - 9 } & Mice & Cockroach & Mosquito & Fly & Surface water (\%) (Eq. 4) & Average temperature $\left({ }^{\circ}\right.$ C) & Rainfall (mm) \\
\hline 2011 & 0.3 & 0.7 & 2.1 & 3.5 & 36 & 15.4 & 1016.8 \\
\hline 2012 & 0.4 & 1.0 & 3.7 & 3.7 & 43 & 15.5 & 1027.1 \\
\hline 2013 & 0.5 & 1.0 & 1.9 & 4.6 & 46 & 16.1 & 882.4 \\
\hline 2014 & 0.4 & 1.3 & 2.3 & 3.6 & 46 & 15.9 & 1083.3 \\
\hline 2015 & 0.3 & 1.0 & 2.5 & 3.7 & 48 & 15.9 & 1371.1 \\
\hline 2016 & 0.3 & 0.7 & 1.4 & 4.6 & 68 & 16.3 & 1612.3 \\
\hline 2017 & 0.4 & 0.7 & 1.9 & 4.0 & 71 & 16.6 & 1109.6 \\
\hline 2018 & 0.3 & 0.5 & 1.8 & 3.7 & 68 & 16.5 & 1145.7 \\
\hline 2019 & ND & ND & ND & ND & 78 & ND & 798.5 \\
\hline
\end{tabular}

Table 2. Vector density, compliance rate of surface water quality, and average temperature in Jiangsu Province for each year during 2011-2019. ND no data available.

\begin{tabular}{|c|c|c|c|c|c|c|c|c|c|c|c|c|c|c|c|}
\hline \multirow[b]{2}{*}{ Variables } & \multicolumn{3}{|c|}{ Type A and B } & \multicolumn{3}{|c|}{ Viral hepatitis } & \multicolumn{3}{|c|}{ Dysentery } & \multicolumn{3}{|c|}{ Typhoid and paratyphoid } & \multicolumn{3}{|c|}{ Other infectious diarrhea } \\
\hline & $\beta$ & SE & $p$ value & $\beta$ & SE & $p$ value & $\beta$ & SE & $p$ value & $\beta$ & SE & $p$ value & $\beta$ & SE & $p$ value \\
\hline $\begin{array}{l}\text { Accumulative house- } \\
\text { holds using sanitary } \\
\text { toilets }\end{array}$ & -0.060 & 0.009 & 0.001 & -0.024 & 0.006 & 0.008 & -0.036 & 0.004 & $<0.001$ & -0.001 & 0.000 & 0.188 & 0.043 & 0.032 & 0.228 \\
\hline $\begin{array}{l}\text { Accumulative house- } \\
\text { holds using harmless } \\
\text { sanitary toilets }\end{array}$ & -0.025 & 0.002 & $<0.001$ & -0.010 & 0.002 & 0.001 & -0.015 & 0.001 & $<0.001$ & 0.000 & 0.000 & 0.305 & 0.021 & 0.012 & 0.131 \\
\hline $\begin{array}{l}\text { Accumulative house- } \\
\text { holds using sanitary } \\
\text { public toilets }\end{array}$ & -0.429 & 0.056 & 0.001 & -0.167 & 0.041 & 0.010 & -0.260 & 0.027 & $<0.001$ & -0.003 & 0.004 & 0.550 & 0.417 & 0.208 & 0.101 \\
\hline Density of mice & 19.437 & 28.123 & 0.515 & 13.291 & 11.521 & 0.293 & 6.190 & 16.715 & 0.724 & -0.074 & 0.636 & 0.911 & -15.989 & 40.059 & 0.704 \\
\hline Density of cockroach & 6.006 & 5.402 & 0.309 & 3.704 & 2.097 & 0.128 & 2.362 & 3.294 & 0.500 & -0.063 & 0.127 & 0.643 & -12.165 & 6.575 & 0.114 \\
\hline Density of mosquito & 2.381 & 1.990 & 0.277 & 1.013 & 0.872 & 0.290 & 1.401 & 1.146 & 0.267 & -0.033 & 0.046 & 0.506 & -2.203 & 2.942 & 0.482 \\
\hline Density of fly & -2.369 & 3.205 & 0.488 & -0.798 & 1.422 & 0.595 & -1.660 & 1.814 & 0.396 & 0.083 & 0.065 & 0.247 & 0.129 & 4.650 & 0.979 \\
\hline $\begin{array}{l}\text { Surface water (meets } \\
\text { Class III standards and } \\
\text { above) }\end{array}$ & -0.239 & 0.033 & $<0.001$ & -0.104 & 0.012 & $<0.001$ & -0.126 & 0.024 & 0.001 & -0.001 & 0.002 & 0.588 & 0.337 & 0.084 & 0.005 \\
\hline Average temperature & -7.778 & 1.351 & 0.001 & -3.201 & 0.745 & 0.005 & -4.523 & 0.759 & 0.001 & -0.054 & 0.072 & 0.483 & 9.123 & 3.017 & 0.023 \\
\hline Maximum temperature & -0.731 & 1.039 & 0.508 & -0.267 & 0.458 & 0.581 & -0.464 & 0.596 & 0.466 & -0.001 & 0.024 & 0.983 & 2.197 & 1.204 & 0.118 \\
\hline Minimum temperature & -1.386 & 0.714 & 0.100 & -0.483 & 0.345 & 0.211 & -0.873 & 0.388 & 0.065 & -0.030 & 0.016 & 0.109 & -0.001 & 0.001 & 0.515 \\
\hline Rainfall & -0.003 & 0.006 & 0.676 & -0.002 & 0.002 & 0.505 & -0.001 & 0.003 & 0.692 & 0.000 & 0.000 & 0.317 & -0.009 & 0.009 & 0.337 \\
\hline
\end{tabular}

Table 3. Association between the aggregate annual incidence of Type A and B intestinal infectious diseases and various factors in Jiangsu Province. Bold characters signify Sig at $\mathrm{p}<0.2$. 


\begin{tabular}{|c|c|c|c|c|c|}
\hline Variables & $\beta$ & SE & $p$-value & $95 \% \mathrm{CI}$ & $R^{2}$ \\
\hline \multicolumn{6}{|l|}{ Type $A$ and $B$} \\
\hline Accumulative households using sanitary toilets & -0.036 & 0.003 & $<0.001$ & $-0.043,-0.029$ & \multirow{2}{*}{0.996} \\
\hline Surface water (Meets Class III standards and above) & -0.135 & 0.011 & $<0.001$ & $-0.164,-0.107$ & \\
\hline \multicolumn{6}{|l|}{ Dysentery } \\
\hline Accumulative households using sanitary toilets & -0.016 & 0.002 & 0.001 & $-0.02,-0.012$ & \multirow{2}{*}{0.998} \\
\hline Accumulative households using sanitary public toilets & -0.159 & 0.011 & $<0.001$ & $-0.191,-0.128$ & \\
\hline
\end{tabular}

Table 4. Multiple linear regression models for intestinal infectious diseases in Jiangsu Province.

\begin{tabular}{|c|c|c|c|c|}
\hline Variables & $\beta$ & SE & $p$ value & Adjusted $R^{2}$ \\
\hline \multicolumn{5}{|l|}{ Nanjing City } \\
\hline Average temperature & -5.091 & 1.756 & 0.034 & \multirow{2}{*}{0.860} \\
\hline Population density & -0.023 & 0.007 & 0.019 & \\
\hline \multicolumn{5}{|l|}{ Suzhou City } \\
\hline Density of mosquito & 1.965 & 0.211 & 0.001 & \multirow{3}{*}{0.984} \\
\hline Density of fly & -0.538 & 0.139 & 0.018 & \\
\hline Population density & -0.011 & 0.001 & 0.000 & \\
\hline \multicolumn{5}{|l|}{ Nantong City } \\
\hline Average temperature & -4.692 & 0.705 & 0.001 & \multirow{2}{*}{0.973} \\
\hline Population density & 0.018 & 0.003 & 0.003 & \\
\hline \multicolumn{5}{|l|}{ Huaian City } \\
\hline Density of mosquito & -0.675 & 0.223 & 0.029 & \multirow{2}{*}{0.742} \\
\hline Population density & 0.050 & 0.016 & 0.024 & \\
\hline \multicolumn{5}{|l|}{ Yangzhou City } \\
\hline Density of mice & 11.866 & 2.312 & 0.007 & \multirow{3}{*}{0.966} \\
\hline Average temperature & -1.919 & 0.462 & 0.014 & \\
\hline Minimum temperature & -0.531 & 0.171 & 0.036 & \\
\hline \multicolumn{5}{|l|}{ Zhenjiang City } \\
\hline Density of fly & 6.382 & 1.468 & 0.007 & \multirow{3}{*}{0.851} \\
\hline Average temperature & -22.644 & 4.259 & 0.003 & \\
\hline Population density & 0.500 & 0.115 & 0.007 & \\
\hline \multicolumn{5}{|l|}{ Taizhou City } \\
\hline Density of mice & 1.533 & 0.179 & 0.000 & \multirow{2}{*}{0.934} \\
\hline Density of mosquito & 9.268 & 1.172 & 0.001 & \\
\hline
\end{tabular}

Table 5. Association between the aggregate annual incidence of Type A and B intestinal infectious diseases and various factors in each city. Only significant models with $p<0.05$ are shown in the table.

A city-specific multiple regression analysis was performed. Table 5 presents the statistically significant models for only certain cities. Average temperature was the common variable that was significantly associated with the aggregate annual incidence of Type A and B intestinal infectious diseases, and it showed a negative association with the aggregate annual incidence of Type A and B intestinal infectious diseases in Nanjing $(\beta=-5.091)$, Nantong $(\beta=-4.692)$, Yangzhou $(\beta=-1.919)$, and Zhenjiang $(\beta=-22.644 ; p<0.05)$.

\section{Discussion}

Intestinal infectious diseases remain a health problem in China, particularly in rural areas. The results of the current study highlight the trends of the incidence of various intestinal infectious diseases from 2011 to 2019 in Jiangsu Province and its cities. Although the aggregate annual incidence of Type A and B intestinal infectious diseases was relatively low, their declining trend still warranted observation. This result was comparable with that of the studies conducted in other provinces ${ }^{20,21}$, particularly in Zhejiang Province, the province adjacent to Jiangsu Province 22 .

The annual incidence of Type $\mathrm{C}$ intestinal infectious diseases was much higher than that of Type A and $\mathrm{B}$ combined together. This can be attributed to the presence of many kinds of pathogens of Type C intestinal infectious diseases and the improvement of diagnostic tools and monitoring network ${ }^{23}$. Another reason is that the government had implemented stricter supervision, reporting, and management for Type A and B intestinal infectious diseases, because it leads more serious health effects than Type $\mathrm{C}$ diseases. Epidemiological investigations were conducted in the shortest time to prevent further spreading of the epidemic ${ }^{24}$. The annual incidence of Type C diseases was found to increase year after year, consistent with the results of a study conducted in the 
Lanzhou city of Gansu Province ${ }^{25}$. Among the different Type C diseases, HFMD disease was the most common one. The annual incidence of this disease was found to peak every other year, similar to the distribution of HFMD reported in studies conducted in other regions of China ${ }^{26,27}$. The annual incidence pattern of Type $\mathrm{C}$ diseases observed may be associated with the human immune system. A study indicated the presence of a widespread latent infection in the population. The duration of specific antibodies after getting infected is $>6$ months; however, the specific timeframe needs to be further examined ${ }^{28}$. In addition, children aged $<5$ years were the most affected by the HFMD. The infant antibody positive rate with 6 months is approximately $30 \%$, and it drops to approximately $10 \%$ after $6-12$ months. Subsequently, the antibody positive rate can increase by approximately $12 \%$ per year and stabilize by the age of $5^{29}$.

During the study period, the distribution of diseases in Jiangsu Province were more clustered in the coastal cities as observed in other regions. The cluster of incidences may be related to the unqualified water quality and the dietary intake of the local residents, such as raw or semi-alive seafood ${ }^{30}$. The internal migration of population to the economically developed areas in the eastern and southern parts of the province could be another reason. However, the cluster was not observed in Suzhou city, the southeastern city of Jiangsu Province. This could be because the economic conditions, urbanization levels, healthcare services, and sanitary conditions of this city is well developed ${ }^{31}$. The high aggregate annual incidence rate of Type A and B intestinal infectious disease in certain cities (i.e., Xuzhou, Suqian, Yancheng, Changzhou, and Nantong) can be attributed to flood disasters. Flood-affected areas experience dramatic changes in environmental conditions, including temperature, rainfall, and humidity. These changes affect the number of pathogens and disease vectors, leading to a higher incidence compared with that in nonaffected areas ${ }^{32}$.

The results of the analysis clearly revealed that increase in sanitary toilet coverage rate led to a decline in the incidence of intestinal infectious diseases in Jiangsu Province. This decline was particularly conspicuous for Type A and B intestinal infectious diseases and dysentery. The popularity of sanitary toilets had clear effects on disease prevention, consistent with the results of another study ${ }^{33}$. A study conducted in Yuxi City, Yunnan Province reported that typhoid and paratyphoid continued to occur frequently because of the inappropriate construction of a flushing and sewage discharge system ${ }^{34}$. Therefore, great attention must be focused on during the construction of toilets, particularly sewer pipes that need to be built according to local conditions. Furthermore, human excreta should be regularly cleaned and protective measures should be taken against water source contamination. These interventions can effectively reduce the density of fly maggots - the dominant vector of intestinal infectious diseases ${ }^{35}$.

This study discovered that toilet improvement was not effective in controlling the most common Type C intestinal infectious diseases. This finding is consistent with that of studies conducted in other provinces and cities in China ${ }^{36,37}$. Therefore, prevention and disease control measures should focus on Type $\mathrm{C}$ intestinal infectious diseases through upgradation of toilet improvement technology. Although no positive results have been observed in relevant studies, according to news reports, the incidence of HFMD has been effectively controlled in the Zibo city of Shandong Province and other areas after toilet improvement ${ }^{38}$. Therefore, Enhancing regional communication and sharing experience may be an effective way to improve the effectiveness of toilet improvement.

In addition to the coverage rate of sanitary toilets, the compliance rate of surface water quality was associated with intestinal infectious diseases incidence rate. Surface water is the main source of drinking water. In general, high water quality can lower the risk of intestinal infections. Although people do not drink water from natural sources directly, opportunities for direct and indirect contact with these water sources still exist, such as when water is used for sediment for agricultural purpose, particularly in vegetable fields. The contamination can transfer to human pathogens and result in diseases ${ }^{39}$. Furthermore, water quality was positively associated with other infectious diarrhea. This result was comparable with a study conducted in Suzhou that indicated that the transmission routes of other infectious diarrhea are more complicated (e.g., air droplets and close contact). The existing disinfection technology may not be able to effectively control certain pathogens, leading to higher incidence ${ }^{37}$. Another study identified that drinking habits can also affect the incidence of diseases; that is, residents who drink unboiled water are more likely to get sick compared with those who boil water before drinking ${ }^{40}$. This may explain the association between water quality and other infectious diarrhea incidence.

The impact of meteorological factors on pathogens, hosts, vectors, and susceptible people have been reported. In general, temperature affects the replication and survival of pathogens in the environment, in addition to people's living habits ${ }^{41}$. This implies that the rise of temperature will increase the incidence of certain diseases. However, this association was not observed in our study at both the province and city levels, as indicated in our final regression models. Although a positive association was anticipated, we found temperature to be negatively associated with Type A and B intestinal infectious diseases. This can be explained by the increasing number of cumulative households using sanitary toilets and improvements in sanitary conditions-both of which play a greater role in controlling diseases. Therefore, the effect of temperature on intestinal infectious diseases was not observed.

While other studies have utilized data from smaller city areas, to our knowledge, our study is a rare analysis that employed data from Jiangsu Province as a whole and each city separately ${ }^{33,42}$. Notably, data on sanitary toilets for 2018 and 2019 in this study were changed on the basis of official information. The current study's analysis results can better reflect the effect of toilet improvement policy implementation on the incidence of intestinal infectious diseases. However, our study had several limitations. First, the immunization data of vaccine preventable diseases were not available. Hepatitis A vaccine has been included in the immunization program implemented by Jiangsu Province government since 2008, whereas hepatitis E vaccine and E71 vaccine for HFMD were optional ${ }^{43}$. Since immunization reduces incidences of targeted infectious diseases, and since the study did not consider vaccination against three of the infectious diseases (hepatitis A, HFMD caused by enterovirus-71, and hepatitis E), the study was unable to determine the independent contribution of vaccination to the observed decline in intestinal infections. Second, information on toilet coverage and incidence of Type C 
intestinal infectious diseases in each of the 13 cities were lacking. Our analysis considered only environmental parameters. Therefore, analysis of spatial distribution and the actual association between toilet coverage rate and the incidence rate of intestinal infectious diseases in each city could not be concluded. Third, our study employed an ecological study design to assess the association between toilet coverage rate and annual incidence of diseases. The lack of information on the sample population's personal characteristics, such as drinking or eating behavior and the toilet-using habits, limited us to perform an analysis at the individual level. Therefore, conclusions and recommendations at the individual level could not be provided. Finally, our study included only the variables that were available (i.e., toilet coverage rate, water quality, and meteorological factors) in the analysis. Various predisposing factors associated with intestinal infectious diseases are still left to be examined. Therefore, further investigation is required, particularly at the individual level.

\section{Conclusion}

The increase in the number of sanitary toilets is significantly related to the decrease in Type A and B intestinal infectious diseases, implying that the popularization of sanitary toilets can directly and effectively prevent and control the incidence of Type A and B intestinal infectious diseases. However, toilet improvement interventions could not effectively control Type $\mathrm{C}$ diseases. Considering the coverage rate of sanitary toilets and harmless sanitary toilets has almost achieved full capacity, new technologies could be implemented in the next phase of the toilet improvement campaign. New construction standards can be proposed on the basis of the control of intestinal infectious diseases by current sanitary toilets. Furthermore, particular attention can be paid to later maintenance work. Hygiene promotion should be strengthened and residents should be encouraged to take the initiative to participate in toilet improvement programs that could benefit intestinal infectious disease prevention and control.

Received: 26 February 2021; Accepted: 8 June 2021

Published online: 17 June 2021

\section{References}

1. Doorduyn, Y., Van Pelt, W. \& Havelaar, A. The burden of infectious intestinal disease (IID) in the community: A survey of selfreported IID in The Netherlands. Epidemiol. Infect. 140(7), 1185-1192 (2012).

2. Adams, N. L. et al. Socioeconomic status and infectious intestinal disease in the community: A longitudinal study (IID2 study). Eur. J. Public Health 28(1), 134-138 (2017).

3. Kyu, H. H. et al. Global and national burden of diseases and injuries among children and adolescents between 1990 and 2013 : Findings from the global burden of disease 2013 study. JAMA Pediatr. 170(3), 267-287 (2016).

4. Ross, A. G. et al. Can we 'WaSH' infectious diseases out of slums?. Int. J. Infect. Dis. 92, 130-132 (2020).

5. Teschke, K. et al. Water and sewage systems, socio-demographics, and duration of residence associated with endemic intestinal infectious diseases: A cohort study. BMC Public Health 10, 767 (2010).

6. Kim, J. H., Cheong, H. K. \& Jeon, B. H. Burden of disease attributable to inadequate drinking water, sanitation, and hygiene in Korea. J. Korean Med. Sci. 33(46), e288 (2018).

7. Na, C. \& Xuefei, J. Analysis on the characteristics of bacterial intestinal infectious disease and its prevention and control measures. Public Health Forum Magaz. 22(05), 676-677 (2018).

8. Yuzhen, W. et al. Summary report on background investigation technology of rural toilets and feces treatment in Jiangsu Province. J. Hygiene Res. S3, 34-38 (1995).

9. Shenlan, S., Sheng, W. \& Chengxing, H. Analysis of the monitoring results of "three cell" sanitary toilet in Rural areas of Changshan County. Chin. J. Health Lab. Technol. 24(04), 562-563 (2014).

10. Zheng, S., Hongbo, L. \& Yale, Z. The current situation, issues and strategies of “Toilet Revolution” in China. Chin. J. Environ. Manag. 10(02), 45-48 (2018).

11. Aziz, F. A. A. et al. Prevalence of and factors associated with diarrhoeal diseases among children under five in Malaysia: A crosssectional study 2016. BMC Public Health 18(1), 1363 (2018).

12. Hua, G. et al. Epidemiological trend of typhoid and paratyphoid fevers in Zhejiang Province, China from 1953 to 2014 . Int. J. Environ. Res. Public Health 15(11), 2427 (2018).

13. Prasad, N. et al. Epidemiology and risk factors for typhoid fever in Central Division, Fiji, 2014-2017: A case-control study. PLoS Neglect. Trop. Diseases 12(6), e0006571 (2018).

14. Bin, Z. \& Ying, M. Analysis on the epidemic trend and temporal and spatial clustering of intestinal infectious diseases in China from 2005 to 2015. Chin. J. Public Health 34(08), 1120-1124 (2018).

15. Yaxin, S., Zijie, S. \& Hong, Z. Why should China carry out “toilet revolution"? Available from: http://politics.people.com.cn/n1/ 2019/0919/c429373-31362516.html. Accessed 3 Nov 2020 (2019).

16. Hua, W. The course, Achievement and Prospect of the Patriotic Health Movement-Commemorating the 60th anniversary of the Patriotic health Movement in Jiangsu province. Jiangsu Health Care 14(04), 1-3 (2012).

17. People's Tribune. The main course of toilet reform in China. People's Tribune (Chinese). (10), 115 Article in Chinese available from: https://kns.cnki.net/KXReader/Detail?TIMESTAMP=637589303985686953\&DBCODE=CJFD\&TABLEName=CJFDLAST2018\& FileName $=$ RMLT201810058\&RESULT $=1 \&$ SIGN $=$ zkAb57Hz2Sbj75\%2fZu\%2fZWxfXiVac\%3d (2018).

18. Yongsheng, W., Yansui, L. \& Hualou, L. Regional characteristics and pathway optimization of China's rural toilet improvement. J. Agric. Res. Environ. 36(05), 553-560 (2019).

19. Jiangsu Commission of Health. Jiangsu Commission of Health has released the 2019 provincial statutory report on infectious diseases. Available from: http://wjw.jiangsu.gov.cn/art/2020/5/9/art_49513_9083203.html. Accessed 3 Nov 2020 (2020).

20. Caiyun, L. et al. Epidemioloqical features of type A and B intestinal infectious diseases in Haidian district of Beijing from 2005 to 2016. Chin. J. Prevent. Med. 19(12), 881-885 (2018).

21. Wanmin, W. \& Lihua, G. From 2009 to 2014, Zhengzhou statutory report on the epidemic characteristics of Type A and B intestinal infectious diseases. Occup. Health. 31(19), 2664-2667 (2015).

22. Qifeng, Z. et al. Epidemiological analysis on the category A and B intestinal infectious diseases in Shaoxing city from 2008 to 2018. Modern Prevent. Med. 46(18), 3435-3438 (2019).

23. Zhang, C. et al. Pathogenic characteristics of hand-foot-mouth disease in Hunan Province, 2009-2018. Modern Prevent. Med. 47(09), 1537-1540+1546 (2020). 
24. The National People's Congress of the People's Republic of China. Law of the People's Republic of China on the Prevention and Control of Infectious Diseases. Available from: http://www.npc.gov.cn/npc/c238/202001/099a493d03774811b058f0f0ece38078. shtml. Accessed 3 Nov 2020 (2020).

25. Xiaogin, Y. et al. Epidemiological characteristics of other infectious diarrheal diseases in Lanzhou during 2006 and 2017. Chin. J. Prevent. Med. 20(07), 569-573 (2019).

26. Lin, C. et al. Epidemiological characteristics of hand, foot and mouth disease in Beihai city 2008-2018. Pract. Prevent. Med. 27(04), $438-441$ (2020).

27. Gaoqin, H. \& Maolin, Y. Epidemiology and pathogenic characteristics of hand-foot-mouth disease in Liangping District, Chongqing, 2010 2018. Med. Inform. 33(09), 131-133 (2020).

28. Dan, S. Serological survey of hand, foot and mouth disease among healthy people in HebeiDistrict, Tianjin City, 2016-2019 Year. Med. Inform. 33(08), 147-149 (2020).

29. Yingying, L. et al. Epidemiology and etiology of hand-foot-mouth disease in Hebei Province, 2011-2015. Chin. J. Disease Control Prevent. 21(02), 151-155 (2017).

30. Yi, C. et al. Epidemiological characteristics of typhoid and paratyphoid fever in coastal areas of high incidence during 1950-2017. Shanghai Prevent. Med. 30(06), 519-522+528 (2018).

31. Mengyue, J. \& Yongfeng, Z. The spatial pattern evolution of floating population in Jiangsu under the background of new urbanization. Modern Business 04, 287-288 (2018).

32. Ying, X. et al. Temporal and spatial distribution and evolution characteristics of major meteorological disasters in Jiangsu Province. Chin. Agric. Sci. Bull. 35(19), 112-119 (2019).

33. Yuqin, P. \& Meixia, Z. Analysis on the effect of toilet improvement and sanitary prevention in rural areas. J. Environ. Health 03, 223-224 (2002).

34. Honggiang, Z. et al. Analysis on epidemic trend of class A and B notifiable infectious diseases and its prevention and control strategies in Yuxi City from 1996-2015. Occup. Health 33(04), 495-501 (2017).

35. Huoquan, R. Speed up efforts to improve rural toilets and care for farmers' health. Jiangsu Health Care 04, 23-24 (2007).

36. $\mathrm{Ni}, \mathrm{W}$. Current Situation and Effect Evaluation of Rural Toilet Improvement in Jilin Province from 2008 to 2011 (Jilin University, 2013).

37. Haibing, Y. et al. The role of patriotic health campaign of control and prevention in regional waterborne infectious diseases. Public Health Prevent. Med. 29(04), 5-9 (2018).

38. People's Daily Shandong Channel. Zibo, Shandong: Dry toilet "change" brightened the hearts of villagers. Available from: https:// www.sohu.com/a/258824271_114731. Accessed 3 Nov 2020 (2018).

39. Yao, H. Discussion on ecological comprehensive control measures for small and medium-sized river courses in rural areas. South China Agric. 14(11), 163+168 (2020).

40. Zhang, L. et al. Relationship between diarrhea and environmental health as well as behavioral habits of rural residents in Sichuan. J. Environ. Hygiene 9(06), 593-597 (2019).

41. Zuhua, R., Weilin, Z. \& Tao, L. Research progress on the influence of meteorological factors on human health. Health Educ. Health Promot. 14(06), 479-482+502 (2019).

42. Haiyang, Z. Assessment on the Implementation of Lavatory Improvement Project in Yuhuan County (Zhejiang University, 2013).

43. Jiangsu Commission of Health. Guiding Opinions on Vaccination of Jiangsu Province Type II Vaccine (2017 Edition). Available from: http://wjw.jiangsu.gov.cn/art/2017/12/5/art_7312_6864264.html. Accessed 26 June 2021 (2017).

\section{Acknowledgements}

This study was partially supported by Chulalongkorn University, Grant for Chula Research Scholar, Ratchadaphiseksomphot Endowment Fund (GCURS 59-06-79-01). The author would like to express our gratitude to the Health Committees of Jiangsu Province and 13 cities for providing secondary data and thoroughly explaining the current situation of toilet improvement in Jiangsu Province.

\section{Author contributions}

T.C. and K.K. equally contributed to the study conception, design, data analysis and drafting of manuscript.

\section{Competing interests}

The authors declare no competing interests.

\section{Additional information}

Supplementary Information The online version contains supplementary material available at https://doi.org/ 10.1038/s41598-021-92291-z.

Correspondence and requests for materials should be addressed to K.K.

Reprints and permissions information is available at www.nature.com/reprints.

Publisher's note Springer Nature remains neutral with regard to jurisdictional claims in published maps and institutional affiliations.

Open Access This article is licensed under a Creative Commons Attribution 4.0 International

License, which permits use, sharing, adaptation, distribution and reproduction in any medium or format, as long as you give appropriate credit to the original author(s) and the source, provide a link to the Creative Commons licence, and indicate if changes were made. The images or other third party material in this article are included in the article's Creative Commons licence, unless indicated otherwise in a credit line to the material. If material is not included in the article's Creative Commons licence and your intended use is not permitted by statutory regulation or exceeds the permitted use, you will need to obtain permission directly from the copyright holder. To view a copy of this licence, visit http://creativecommons.org/licenses/by/4.0/.

(c) The Author(s) 2021 